\title{
Teaching Supplement
}

\section{Argument-Explanation Complementarity and the Structure of Informal Reasoning}

\author{
G. RANDOLPH MAYES \\ Department of Philosophy \\ Mendocino Hall 3028 \\ California State University, Sacramento \\ $6000 \mathrm{~J}$ Street \\ Sacramento, CA 95819 \\ U.S.A. \\ mayesgr@csus.edu
}

\begin{abstract}
Argument and explanation are distinct forms of reasoning with an underappreciated complementary relationship. In this essay I define these terms precisely, identify the mischief that results from conflating them, elucidate their complementary relationship and employ this relationship to provide a fruitful approach to analyzing the logical structure of the common editorial.
\end{abstract}

Resume: Les arguments et les explications sont des raisonnements distincts qui ont une complémentarité peu appréciée. Dans cet essai je définis précisément «argument» et «explication», identifie les difficultés qui surviennent lorsqu'on les confond, décris leur complémentarité, et emploie celle-ci pour avancer une approche fructueuse pour analyser les structures des raisonnements dans des éditoriaux courrants.

Keywords: argument, cause, complementarity, editorial, evidence, explanation.

\section{Introduction}

Competent inquiry requires an intuitive grasp of the difference between argument and explanation, two forms of reasoning which are easily conflated. Mastering this difference also makes it possible to see how arguments and explanations are related. In natural language these forms of reasoning tend to occur together and they normally exhibit an interesting complementarity. This fact is not widely appreciated, nor is the reason for it.

In this paper I will briefly summarize the difference between argument and explanation, and then illustrate their complementary relationship by showing how it is modeled in ordinary reasoning

(C) G. Randolph Mayes. Informal Logic, Vol. 30, No. 1, (2010) pp.

92-111. 
contexts, notably the common editorial. Ultimately my point is to show that when analyzing ordinary reasoning, it is a good idea to do so with the expectation of having to disentangle logically distinct but complementary arguments and explanations. When this expectation is not met, it will sometimes point to a fundamental weakness in the reasoning provided.

\section{The difference between argument and explanation}

Argument and explanation are two different forms of reasoning, so let's begin by being clear on what we mean by that. Reasoning is just the process of making certain statements, which we call reasons, in support of other statements, which we call conclusions. This relationship may be visualized as follows:

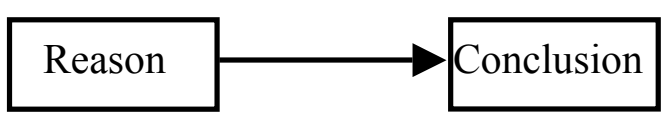

We are called upon to support our statements with reasons whenever a certain kind of question arises with respect to these statements. The difference between arguments and explanations reflects a difference in the kind of question that arises, and the kind of support that the question requires.

Consider a statement like:

(1) The dog has a broken leg.

There are basically two questions that might arise if someone were to make this statement in a context in which its basic meaning is clear to you. On the one hand, you may have little or no prior reason for believing this statement to be true. In this case, your cognitive response may be one of doubt, which you will articulate with a question like: How do you know? This is a request for evidence. Evidence is a kind of reason, and the attempt to provide evidence in support of a conclusion is normally called an argument. Argument reasoning may therefore be represented as:

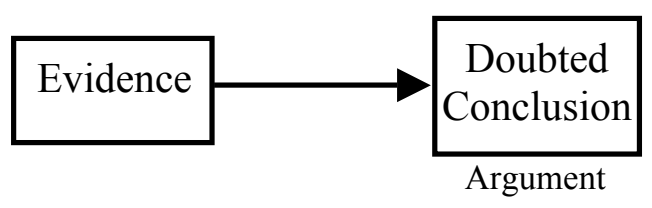




\section{G. Randolph Mayes}

On the other hand, you may already know the dog's leg is broken and hence require no further evidence for the truth of the statement. But you still may not understand why the dog's leg is broken. In this case you will naturally ask something like: How did it happen? This is a request for a cause. Cause is the other kind of reason, and the attempt to provide a cause in support of a conclusion is normally called an explanation. ${ }^{1}$ Explanation reasoning may be represented as:

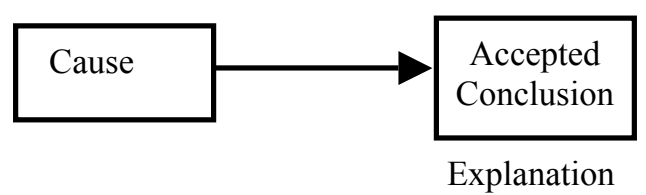

Here is an ordinary language argument for the conclusion that the dog's leg is broken:

(2) The reason I think the dog has a broken leg is that he won't put any weight on it.

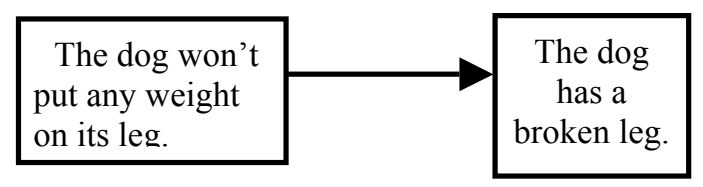

Argument

]

Here is an ordinary language explanation for the same statement:

(3) The reason the dog has a broken leg is that it was hit by a car.

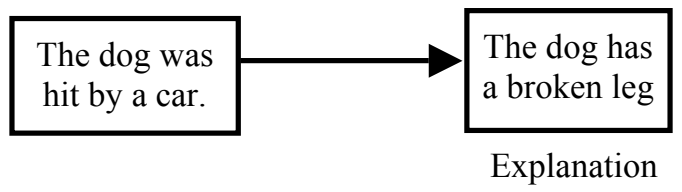

\footnotetext{
${ }^{1}$ In some fields of philosophy, notably the philosophy of mind, it is traditional to make a distinction between a reason and a cause, where reasons are understood to move the mind according to logical principles as opposed to causal ones. We are not following this usage here. For us, a reason is just any statement given in support of a conclusion. Some reasons identify evidence, others identify cause.
} 
It is also interesting to note here that without the phrase "the reason that" or various other possible reasoning indicators, it is not possible to distinguish an argument from an explanation. For example, consider:

(3') The dog's leg is broken. It was hit by a car.

This might be intended as an explanation of the fact that the dog's leg is broken. But it might also be offered as an argument that the dog was hit by a car, with the broken leg serving as evidence. This means that whether reasoning is understood to be providing evidential or causal support depends crucially on the context of utterance. These relations are not, like logical entailment, formal relations between the propositions expressed by the corresponding statements.

In summary, then, an argument is a piece of reasoning in which the reason is intended to provide evidence for accepting a doubted conclusion. An explanation is a piece of reasoning in which the reason is intended to provide a cause for an already accepted conclusion.

It is sometimes said that rational inquiry aims at two things: knowledge and understanding. We can now say that argument and explanation are the reasoning tools that we use to accomplish these two goals. Argument attempts to establish knowledge by giving evidence that reduces doubt. Explanation attempts to establish understanding by supplying causal connections between accepted facts.

\section{The distinction elaborated}

There are a few points worth elaborating before proceeding.

First, we note that several distinct reasons can be given for a single conclusion, as well as reasons for the reasons themselves. For example, the following ordinary language explanation is represented by the diagram below.

(4) Frank is in a bad mood both because he had a bad day at work and because his son Mike didn't do his chores. Mike was at a party last night and ended up sleeping all day. Plus the kid is just lazy. 


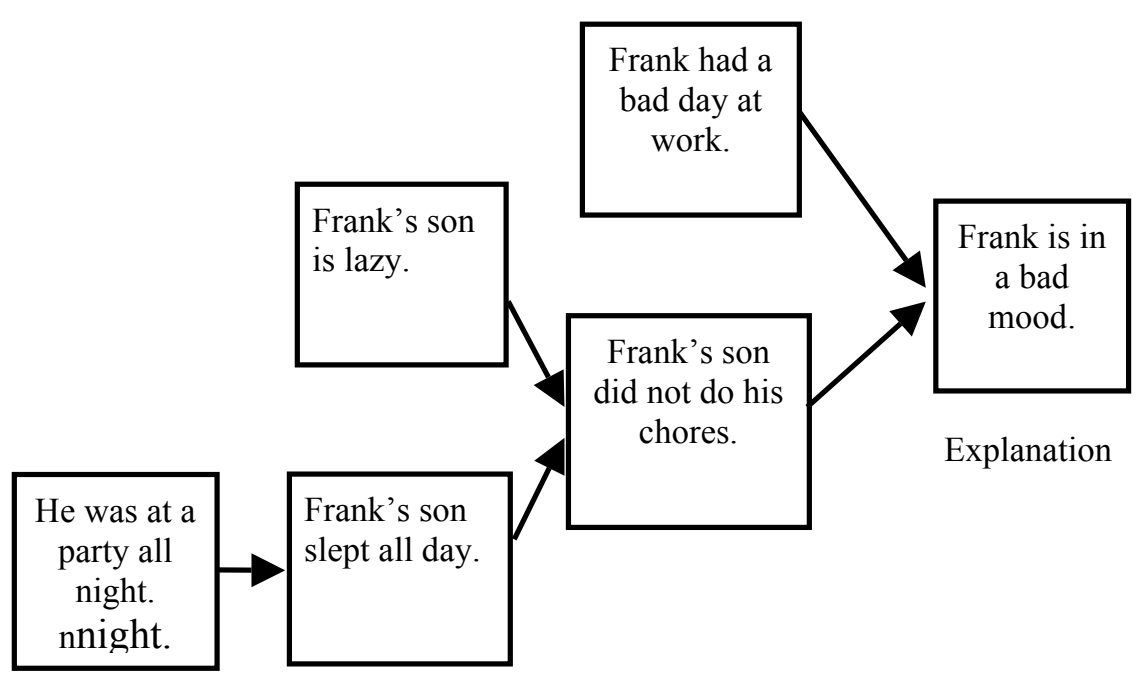

Second, we emphasize that since argument and explanation have different functions, it is essential to analyze them as separate structures. Consider the following example and a proposed reconstruction.

(5) The baby has been crying all day because she is sick. Her temperature is $103^{\circ}$. I think she has must have some kind of infection.

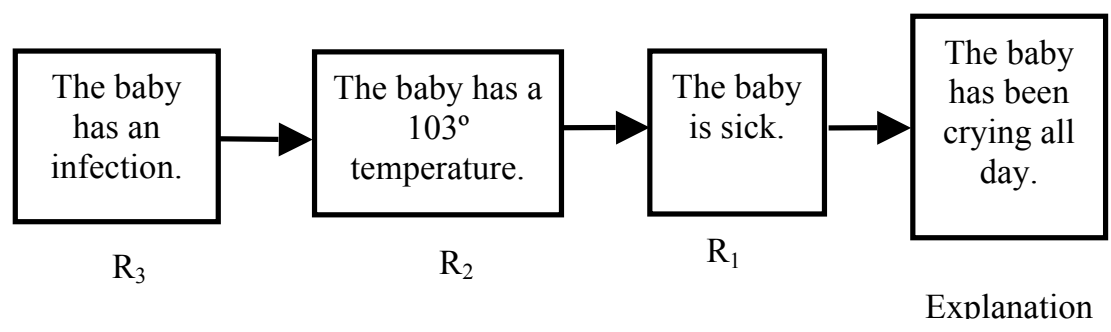

Because we have identified this reasoning as an explanation of the fact that the baby is crying, every reason in the reconstruction above must be read as a cause of the reason or conclusion that it supports. But notice that this relationship does not hold between $\mathrm{R}_{2}$ and $\mathrm{R}_{1}$ : the baby's temperature is given as evidence that the baby is sick, not a cause of the baby's sickness. Hence, this reconstruction is faulty. The argument is properly reconstructed as a distinct argument and explanation. Here is one of several acceptable reconstructions: 


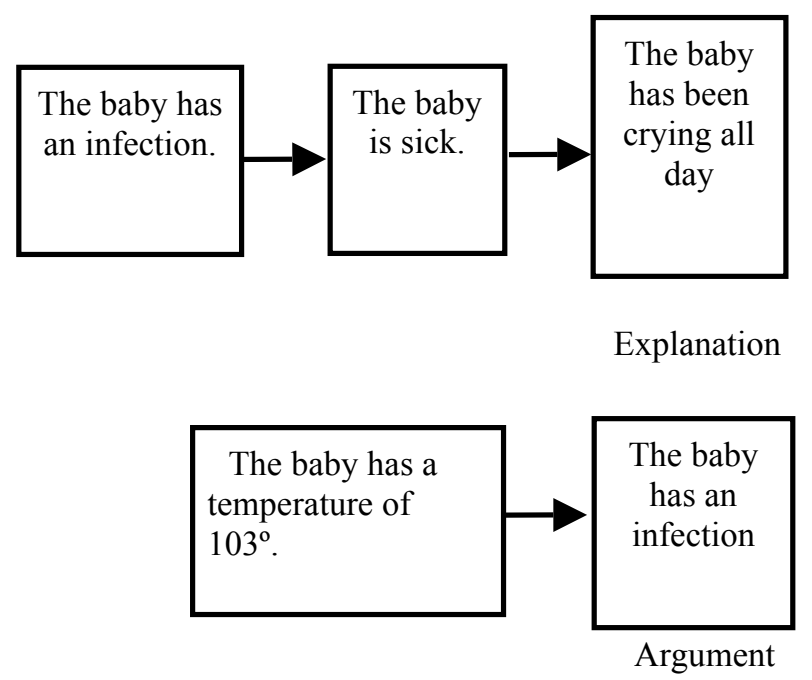

Third, as noted briefly in the preceding section, it is important to understand that whether a piece of reasoning is properly reconstructed as an argument or an explanation depends on the assumptions of the individual who has produced the reasoning. To further understand the importance of this point, consider the following example.

(6) The reason poverty is rising worldwide is that the global economy has made it impossible for countries to provide stable working conditions for its citizens.

This is easily recognized as an explanation by anyone who does not have a view about the prevalence of worldwide poverty. The problem arises for someone who does. If, for example, you know that poverty has in fact been declining worldwide for a very long time, then you automatically doubt the conclusion that poverty is rising. If you doubt this conclusion, it is reasonable for you to request an argument in support of it. However, this does not mean that it is legitimate to interpret the reasoning above as such an argument. In the reasoning above the author clearly (if incorrectly) accepts the conclusion that poverty is rising worldwide, and he provides a cause of this alleged fact. So, it is properly interpreted as an explanation, even if you do not accept the conclusion.

The assumptions of the author also determine whether a piece of reasoning is best interpreted as an argument. Although the author of an argument often already does accept the argument's conclusion, the social function of the argument is to convince 
others. So the assumption that matters here is the assumption that others do not accept the conclusion. Of course, sometimes this assumption is false. Just as people often inappropriately explain conclusions that others do not accept, they sometimes unnecessarily provide arguments for conclusions that others already do accept.

Of course, the real world does not conform to this tidy account. Since people are not generally proficient in the distinction between argument and explanation, it will sometimes be necessary to claim that while a person may think he has provided evidence or cause, in fact he has not. We will have a little more to say about this below.

Fourth, our distinction between argument and explanation is a technical one, and it does not capture all of our ordinary intuitions about the meaning of these terms. Obviously it is not intended to capture the ordinary sense of 'argument' as a debate or verbal battle. More importantly, however, it does not capture a common way of using the term 'explanation' in normative contexts. For example, it is very common to say things like:

(7) Sarah explained to Annie that she shouldn't talk back to the teacher because it makes the teacher angry.

In this context it is clear that Sarah assumes that Annie does not accept the conclusion that she should refrain from talking back to her teacher, and Sarah has provided a reason for Annie to accept it. So we would reconstruct this as an argument, even though the term explanation is used here in an intuitively acceptable way.

Finally, there are several things about the above exposition that may be disturbing to those with some knowledge of formal logic or the philosophy of science. These primarily concern things I have said above that may appear to involve confusions concerning the nature of argument, explanation, causation and logical implication. I do not want to discuss any of these issues here, because I think it would be distracting to the typical reader, but I have dealt with them in the following footnote. ${ }^{2}$ (As a philosopher of science

\footnotetext{
${ }^{2}$ I note here a few legitimate concerns that might be troublesome to those with some knowledge of logic and philosophy of science.

First, the reasoning diagrams I am using here clearly are not intended to represent deductively valid reasoning patterns. This method can be easily adapted to such representation by thinking of each arrow as indicating the existence of a principle (classically, the major premise in a Barbara syllogism) that would secure a deductive relationship between a reason and the reason or
} 
myself, and someone who is familiar with accepted terminology, I simply ask the sophisticated reader to give me the benefit of the doubt here.)

\section{Functionally Related Arguments and Explanations}

Consider the following piece of natural language reasoning and think about how you might reconstruct it. Is it an argument, an explanation, or some combination of the two?

(8) I just failed our final exam. I know it's because only got three hours of sleep last night. I never do well on tests when I don't get enough sleep.

The answer is that it is an explanation followed by an argument. This may be easier to see if we reconstruct it as a dialogue.

Serge: I failed the final exam.

Fran: Really? Why? I didn't think it was that hard.

Serge: It's because I only slept three hours last night.

Fran: How do you know it's because of that? Maybe you just didn't study enough.

Serge: No, it's not that! I just never do well on anything when I don't get enough sleep.

conclusion it is supporting. I exclude this from the general discussion for the sake of simplicity.

Second, there is a bit of ambiguity in the way I am using the terms 'cause' and "evidence". It is possible to think of evidence in both metaphysical and linguistic terms. For example, we can say that smoke is evidence of fire, and we can say that the statement "there is smoke" is evidence for the statement "there is fire". This does not apply so well to the term 'cause,' however. We can say that fire causes smoke, but it is very strange to say that the sentence "there is fire" is a cause of the sentence "there is smoke". Nevertheless, a careful reading of my explication of the distinction between argument and explanation will show that I am using the term in this way. Again, I do so for simplicity's sake only. When I say that the reason provides a cause of the conclusion, what I mean is that the reason represents a cause of the fact represented by the conclusion.

Third, I use the term 'cause' very generally, and do not restrict the term to its spatiotemporal sense. There are a variety of synchronic explanatory relationships in mathematics, logic, and science that are permitted by my use of the term. 
We can represent this reasoning as follows.

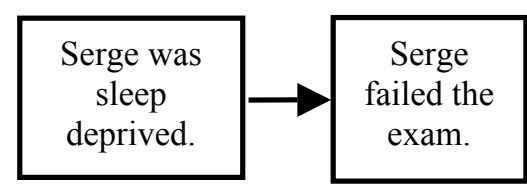

Explanati

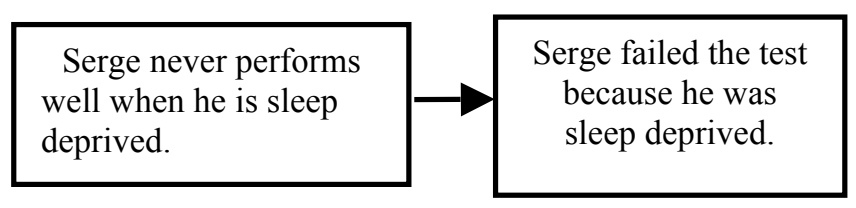

Argument

Serge, then, has proposed a cause, hence an explanation, of his poor test performance. But he also has anticipated Fran's skepticism, specifically in regard to the explanation itself, and provided an inductive argument supporting his statement that his poor performance is the result of sleep deprivation.

Arguments with causal statements in their conclusions are called causal arguments. They are not explanations, but arguments given in support of a certain explanatory relationship. Those who have been trained to think of reasoning as essentially argumentative in nature may be inclined to suggest that the causal argument above exhausts all the reasoning performed in this example But this is a mistake, since it commits us to the obviously false claim that Serge intends no logical relationship between the first two statements of (8).

Now consider the following example:

(9) I wish the coach would stop running that play. It hardly ever works. He only does it because fans love it when it works.

We can render this as a dialogue as well:

Barb. I wish the coach would stop running that play.

Butch: What makes you say that? I like it.

Barb: It hardly ever works!

Butch: Well why does she call it then?

Barb: Because fans like you like it. 
This can be analyzed as follows:
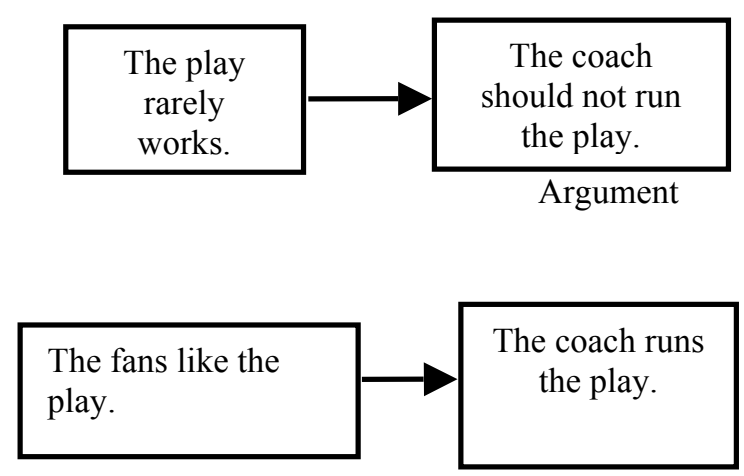

Explanation

As noted in the previous section, it would be intuitive to describe this as "Barb explaining why the coach should not run the play." Clearly, however, this reasoning is an argument, since the play not working is provided as evidence that it shouldn't be run. The explanation itself arises in answer to a question that is actually generated by the argument. Why would a coach run a play that doesn't work? In the absence of a plausible answer to this question, the argument itself remains dubious.

Note that nothing I have said rests on evaluations of the reasoning provided in these examples. However, it is important to see that we cannot properly evaluate this reasoning in the absence of an understanding of its structure. Consider what a reconstruction of this reasoning might look like if we artificially restricted ourselves to the concept of argument.

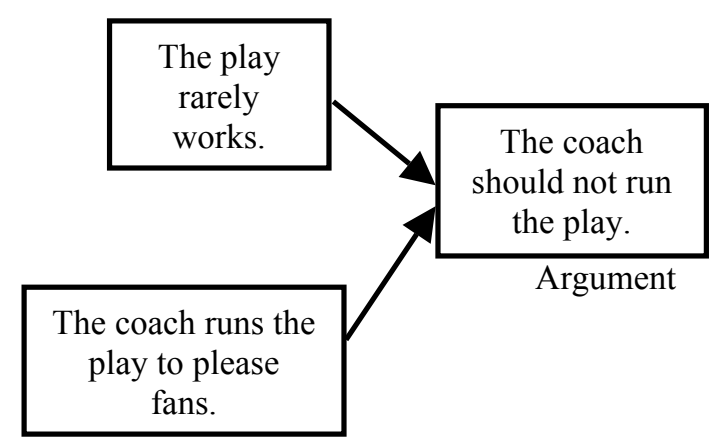

Here we have reconstructed the explanation as a causal statement and represented this statement as evidence that the coach should not run the play. But this is not a particularly charitable interpret- 
ation, as the causal statement actually adds nothing at all to the strength of the argument. Whether or not a play should be used depends entirely on its expected effects, not what a coach's ultimate motivations may be in using it. So this procrustean reconstruction fails to comprehend the actual purpose of the causal claim; viz., to answer a question that is generated by the argument itself.

\section{The relationship elaborated}

As the examples above suggest, there is an important sense in which arguments and explanations complete each other. To see why this is so, consider the following example:

(10) Diet drinks do not aid in preventing obesity. In fact recent studies have shown that the consumption of diet drinks is positively correlated with weight gain: the more of them you drink, the fatter you get.

This is best reconstructed as an argument that may be represented as follows:

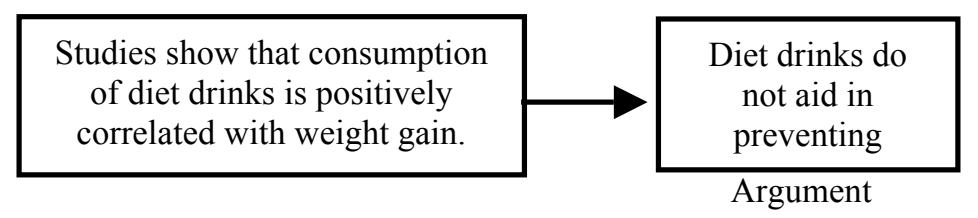

What's interesting about this argument is that the conclusion is highly counterintuitive. It violates a simple causal model according to which weight gain is a function of caloric intake. But notice what happens when the argument is supplemented by the corresponding explanation.

(10') Diet drinks do not aid in preventing obesity. In fact recent studies have shown that the consumption of diet drinks is positively correlated with weight gain: the more you drink, the fatter you get. The problem is that relatively few of our calories actually come from what we drink. People who consume diet sodas with their meals think that they are cutting their caloric intake significantly by doing so, but in fact they raise it believing that their virtuous drinking entitles them to bigger helpings of food.

This explanation employs a different causal model, which allows us to make sense of the conclusion of the argument. In the absence 
of this explanatory model, we find that we remain skeptical of the argument, wondering for example whether the studies are flawed in some way, or whether they are done by researchers with a bias against fat people.

I stress here that explanation does not (and by definition can not) supply any more evidence for the argument's conclusion; but it does help us to accept the argument by allowing us to fit the result into a comprehensible causal pattern. Note also that no argument has been given above in support of this causal model. We are asked to take it at face value, and for all we know the correct explanation is something entirely different. (Perhaps diet drinks actually increase our appetite for sweets.)

Compelling explanations are actually quite dangerous in this way, for they are easily used to persuade people who can detect reasoning, but who can not distinguish between argument and an explanation. For example:

(11) Increasing the minimum wage harms the very people it is intended to help. In order to regain their profit margin employers simply terminate a certain percentage of their lowest paid employees and require those who remain to be more productive. The inevitable result: higher unemployment and poorer working conditions than before.

People without a view on the subject may find themselves persuaded by this reasoning in support of the conclusion that minimum wage laws hurt the poor. After all, the reasoning is clear and compelling:

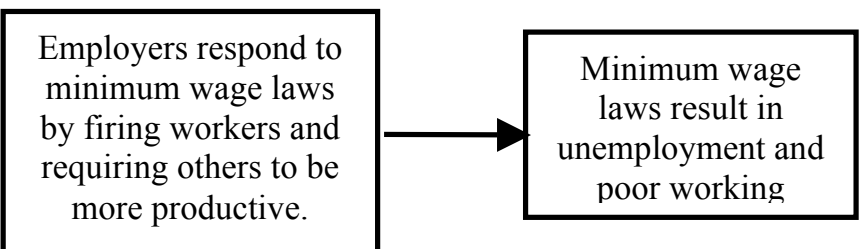

The problem with allowing this reasoning to convince you of the conclusion is that it is not an argument. The reason provides a link in a causal chain. It tells us why minimum wage laws in fact lead to unemployment and poor working conditions. If the reason were functioning as evidence, then it would have to provide data showing a positive correlation between rises in minimum wage and low wage job loss. 
It is worth taking a moment to understand this point fully. Try to think of the reason as evidence. The simplest way to do this is to ask an evidential question of the conclusion: "How do you know that minimum wage laws result in unemployment and poor working conditions?" Now imagine your interlocutor answers:

"I know this because employers simply respond to minimum wage laws by firing workers and requiring others to be more productive." If we seriously think of this as evidence, the reasoning becomes entirely question-begging. If you actually doubt the conclusion, you doubt this reason just as much, for it simply assumes the truth of the conclusion. On the other hand, if you are already inclined to accept the conclusion on the basis of statistical evidence, then this is not a problem. Assuming the truth of the conclusion is just what explanations $d o$. Hence, we should not say that this is simply a question-begging argument. We should say that it is an explanation trying to do an argument's work.

So, arguments are needed to complete explanations just as explanations are needed to complete arguments. Note that the missing argument in the above example is not one that has the explanation itself as a conclusion (as in example [8]). Rather, what is missing is evidence for the actual conclusion of the explanation. Now consider another example:

(12) The president would not admit that it was a mistake for the U.S. to invade Iraq because he is a very proud man who can not admit his mistakes. After two terms in office, we have ample evidence of his hypersensitivity to criticism and his reluctance to seek advice from people who might disagree with him.

Here we have an explanation and a complementary argument. 


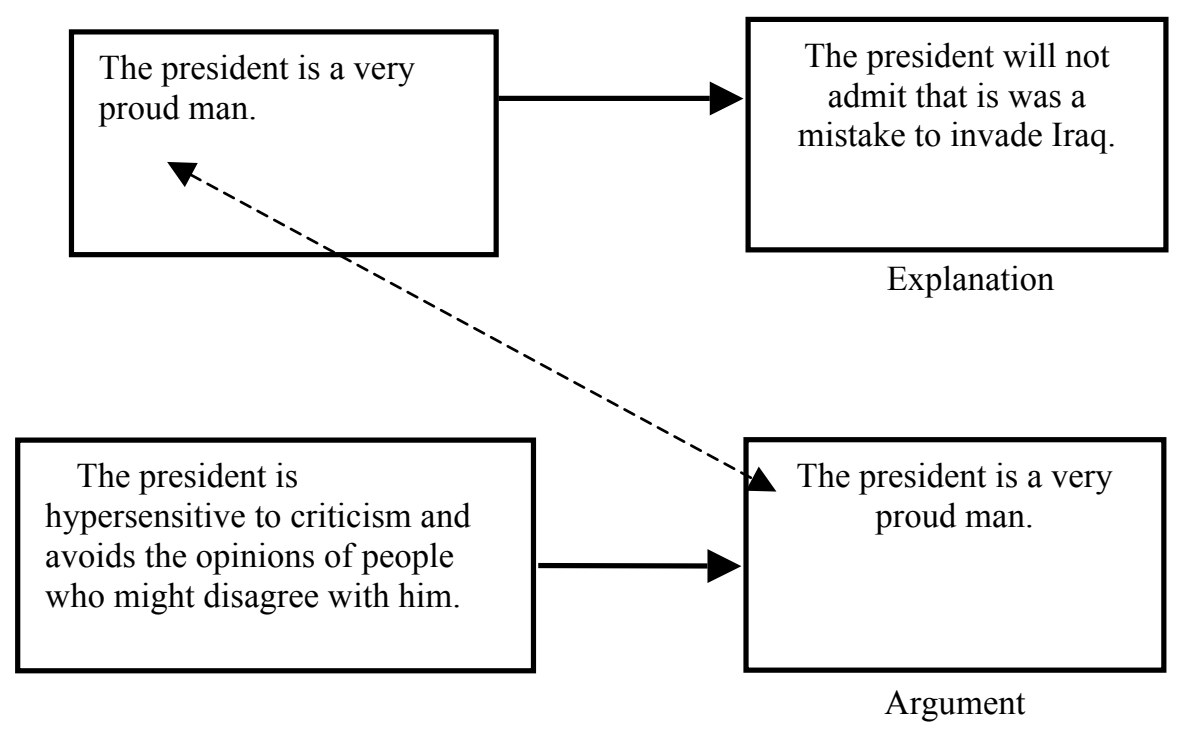

The dashed double arrow above simply indicates that the reason provided in the explanation is identical to the conclusion of the argument. This again is not an argument in support of the causal claim itself. The argument establishes only that the president is proud, not that his pride is what prevents him from admitting that it was a mistake to invade Iraq. (Perhaps what prevents him from admitting the mistake is compelling evidence that it was not a mistake, or belief that admitting the mistake would be counterproductive.)

The simplest way to characterize the complementary relationship between argument and explanation is to return to the distinction between knowledge and understanding. Argument is the mechanism by which we produce knowledge. Explanation is the mechanism by which we produce understanding.

Since even extremely compelling arguments are susceptible to error, and since they often tend to contradict expectations based on beliefs we already hold, we remain skeptical of their conclusions until we are provided with some understanding of how they occur. The circumstantial evidence presented may strongly favor the conclusion that Smith committed the murder, but absent a motive (an explanation of Smith's behavior) we reasonably wonder whether the evidence was reliably collected. The evidence against the existence of a luminiferous aether was compelling, but absent an acceptable causal model for the propagation of light waves, the scientific community reasonably wondered whether the aether was detectable by the methods employed. 
Similarly, the understanding afforded even by ingenious hypotheses must remain suspect until we have some evidence, on the basis of independent argument, that the hypotheses are actually true. Darwin's theory of natural selection, one of the most awesomely explanatory hypotheses in the history of science, was very reasonably doubted for decades in the absence of independent evidence that the earth was of sufficient age to have produced complex life forms by this mechanism. The understanding afforded by a hypothesis may be a reason for preferring it to its known competitors; but it will not be regarded as established knowledge until it successfully predicts previously unobserved phenomena.

\section{An application: the logical structure of editorials}

I conclude this essay by showing that the complementary relationship of argument and explanation is evident in the structure of most editorials. I regard this as an important observation because it means that for the most part when you read an editorial with an eye to understanding the author's reasoning you should not be satisfied simply with representing the arguments put forth in favor of the position being advocated. Rather, you should also be able to say what sorts of complementary explanations the author has provided to repair any tears the argument may have produced in the fabric of your understanding.

Editorials typically derive their interest from providing arguments in support of conclusions that are not generally accepted. They are usually normative conclusions, like:

- Medicare should be privatized.

- Marijuana should not be illegal.

- Homosexuals should not be permitted to marry.

- Torturing terrorists should be permitted.

But sometimes they are just interesting empirical claims that draw no explicitly normative conclusions. For example

- Scientists exaggerate the threat of global warming.

- Charitable giving is selfishly motivated.

- Circumcision prevents AIDS.

- In 20 years robots will do most routine household chores.

Interestingly, most authors display some awareness of our reluctance to accept conclusions, regardless of the evidence provided, if they leave certain explanatory issues are not addressed. The nature of these questions varies somewhat. With respect to 
arguments in support of normative conclusions, the standard explanatory question that arises concerns either the fact that other educated people disagree with the author or the fact that things are not the way the author says they should be. For example:

(13) Weather services should stop reporting the wind chill factor. Wind chill is supposed to be a measure of how cold it feels due to wind, as opposed to how cold it really is. But, as opposed to the actual temperature, how cold the air feels varies dramatically for different body types. In pretending to be an objective measurement, the wind chill factor misleads the public about the actual danger. For example, it suggests that on a day when the temperature is 35 degrees Fahrenheit and the wind chill is -20 they should dress more warmly than on a windless day when the temperature is 28. But no matter how cold it feels, you can not get frostbite on a day when the temperature is above freezing. The truth is that weather services report wind chill, only because it spices up the weather news. Another chilly winter day is barely worth mentioning, but a wind chill of -20 is something to get excited about.

The author of this editorial is clearly arguing for the normative conclusion that weather services should not report wind chill, giving as evidence ways in which wind chill misleads and even endangers the public. But the author also clearly recognizes the importance of anticipating the following skeptical question: If it is so misleading, why do weather services report it? This, of course, is a request for an explanation of the fact that weather services report the wind chill, and it arises because the argument the author is providing conflicts with our general expectation that data reported on a daily basis by news organizations normally has some practical value. By explaining this phenomenon as a marketing tool, he allows us to conceive of the phenomenon in a way that does not contradict this general expectation.

Again, it is important to stress that while providing a complementary explanation may be an important normative requirement, it is easy to lower our resistance to poor arguments by providing specious explanations. This example is a case in point. The critical problem here is really that the evidence provided is only very weak support for the argument's conclusion. Indeed the evidence provided would be stronger support for the conclusion that weather services should simply educate the public about the meaning of wind chill. So here the explanation seems to be making a rather poor argument seem more plausible than it really is. (The use of unsubstantiated explanations to provide specious 
plausibility for weak arguments is a nice technical definition of the common phrase "explaining away.")

Now consider the following example for another normative conclusion:

(14) People generally believe that anabolic steroids should be outlawed in professional sports because they believe that they are dangerous to the athletes who take them and because they think it gives athletes an unfair advantage over others. But both of these reasons are seriously flawed. Of course, it is dangerous to take drugs without medical supervision, but this is what happens when drugs are illegal. Under proper medical supervision the use of steroids can enhance athletic performance, protect athletes from injury, speed recovery from injury, and extend athletic careers. Whatever minor danger exists from taking steroids under medical supervision is nothing compared to the danger of the sport itself. Sure, steroids provide an advantage, but so do genes, physical training, and good coaching. Genetic unfairness is irremediable and it does not interfere at all with our enjoyment of sport. The unfairness of steroids and other advantages can be removed simply by making them available to everyone.

This is an interesting example to consider because it allows us to underscore the importance of the difference between an argument and a psychological explanation of someone's beliefs. Note, first of all, that the author of this passage clearly believes that steroids should be legal, and she provides a clear argument for this conclusion, which we represent schematically below:

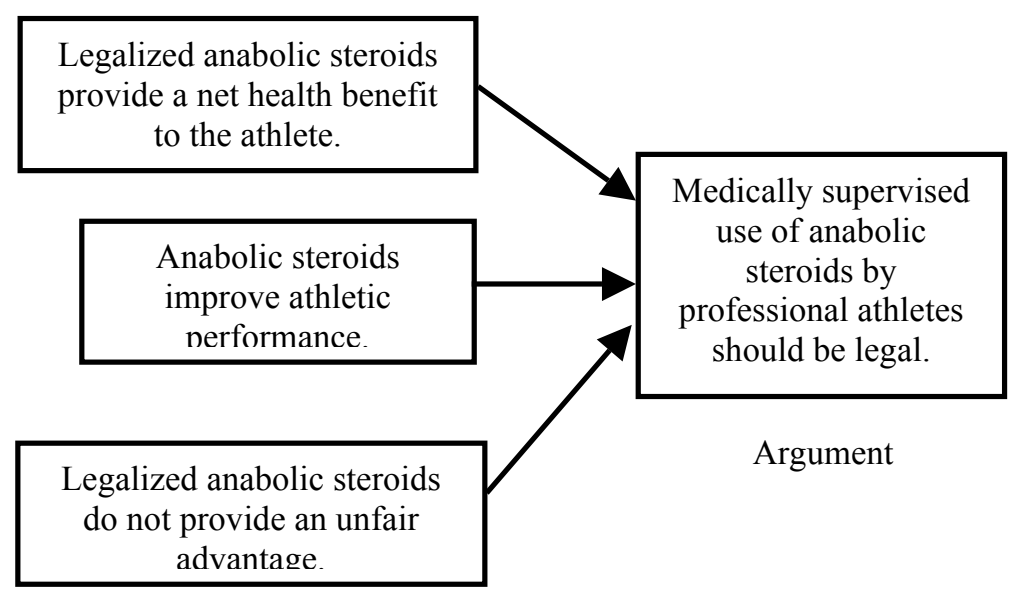

The author begins this editorial by noting reasons given by others for a conclusion that she rejects. Obviously, it would be 
incorrect to represent it as an argument that she is making herself. So, how is it functioning? One way of answering this question is to invoke the use/mention distinction. The author mentions the argument for the purpose of refuting it, but does not actually use it herself. Another approach, however, is to observe that she has offered a psychological explanation of the beliefs of other people, which we represent as follows:

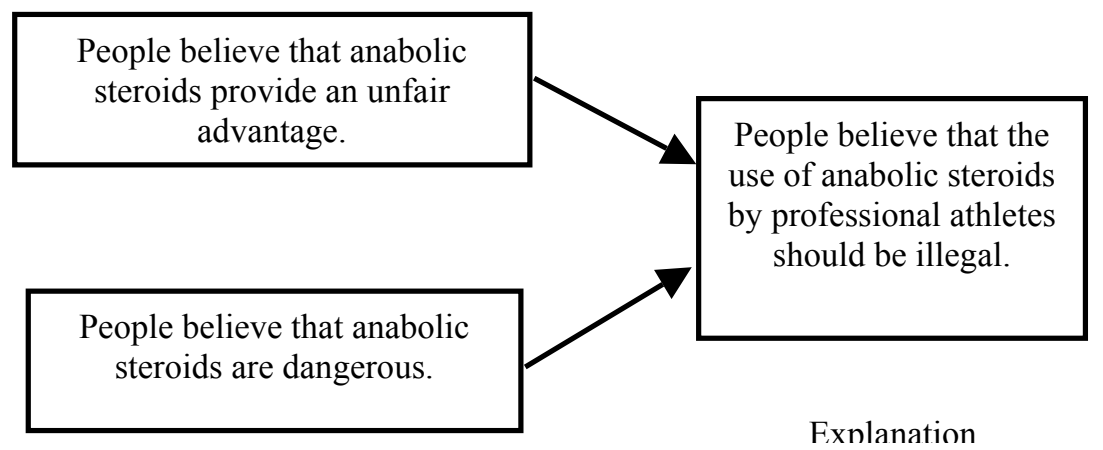

This is an explanation because it represents a causal relationship between beliefs. The fact that people believe anabolic steroids to be dangerous is a causal factor in their belief that they should be illegal. Because the author's argument contains evidence that contradicts the content of these beliefs, this explanation functions to explain the existence of opposing views as the result of error. As in the previous example, the author is anticipating an explanatory question, specifically: "If this is really true, why do so many people believe otherwise?" The author understands that her audience will not accept her reasoning in the absence of some plausible explanation of the fact that so many people disagree with her.

A similar pattern can be observed in editorials advancing empirical claims. The standard pattern is to make an argument for a claim that is not generally known, and then provide a causal model that explains it. (This is actually just the standard idealized pattern of scientific inference: produce experimental data in support of a particular hypothesis, then suggest a model that allows one to both explain the data, and predict the results of future experiments.) Here is a short example:

(15) The astonishing technological advances of the $20^{\text {th }}$ century transfigured the lives of ordinary people. Wealth and life expectancy soared. Things that were once luxuries, like airline travel and phone service, became affordable for everyone. But did the ease and 
convenience of modern life contribute to our happiness? Surprisingly, the answer to this seems to be no. The average levels of happiness reported by Americans in the 1940 's are not much different than they are today. In fact, since the 1950s reports of major depression have increased considerably. There are some exceptions, however. An especially interesting one is the Amish. Their depression rates are very low relative to the rest of society and they report higher general levels of happiness. What makes the Amish interesting, of course, is that they are unique in refusing modern technological conveniences. They shun automobiles, cell phones, televisions, and computers. Amish children do not have iPods, Game Boys or PlayStations. This suggests a very interesting possibility. Perhaps the conveniences of modern technology, instead of making us happier, are actually contributing to human misery. Maybe all the choices and conveniences of modern life that we think are so wonderful ultimately just serve to sever the social bonds that make human life truly meaningful.

Note that in the absence of an expectation of argumentexplanation complementarity one might be tempted to reconstruct this as an argument for the conclusion that technology contributes to human misery as follows:

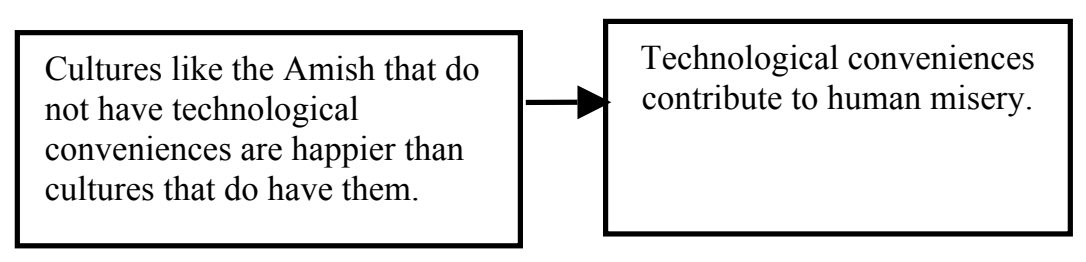

Argument

This is a perfectly comprehensible argument, but of course it is also a very weak argument since it advances a causal claim based on a simple correlation. People do make weak arguments of this sort, but the author of this passage has not done so here. Rather, he has provided an argument for the conclusion that technology has not made us happier, together with an explanation of this fact as follows: 


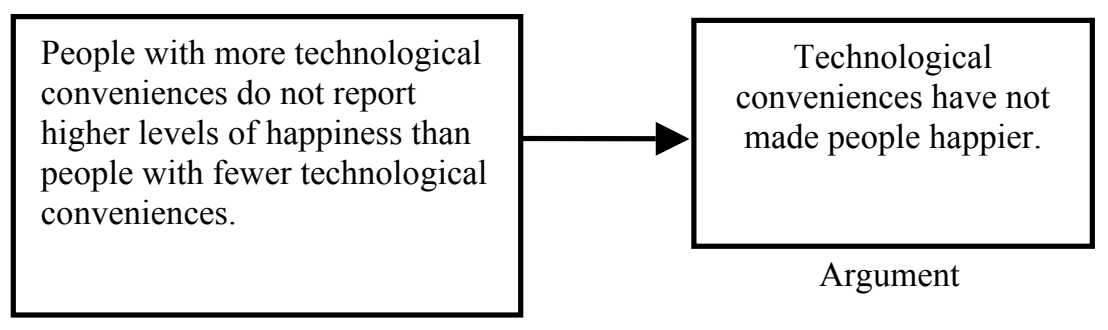

\begin{tabular}{|l|l|}
\hline $\begin{array}{l}\text { Technological convenience } \\
\text { create some conditions of } \\
\text { unhappiness; e.g., social } \\
\text { isolation. }\end{array}$ & $\begin{array}{c}\text { Technological } \\
\text { conveniences have not } \\
\text { made people happier. }\end{array}$ \\
\hline
\end{tabular}

Explanation

This analysis captures the fact that the cause contained in the explanation is presented only as a hypothesis that would explain the data, and it prevents us from making uncharitable accusations of fallacious reasoning.

My experience in reading editorials is that the vast majority of them can be accurately analyzed in one of the above ways. Only rarely do we see argument in the absence of explanation and vice versa. Of course, I have not presented a compelling argument for this view here, since this would require a study in which a large number of randomly collected editorials is examined for this relationship. On the other hand, if I am right, I have at least provided an explanation of this fact: arguments and explanations have a complementary relationship and reasoning is normally perceived as incomplete when one occurs in the absence of the other. 